PROCEEDINGS OF THE

AMERICAN MATHEMATICAL SOCIETY

Volume 130, Number 4, Pages 1043-1054

S 0002-9939(01)06145-7

Article electronically published on September 14, 2001

\title{
A CRITERION FOR CORRECT SOLVABILITY OF THE STURM-LIOUVILLE EQUATION IN THE SPACE $L_{p}(R)$
}

\author{
N. CHERNYAVSKAYA AND L. SHUSTER \\ (Communicated by Carmen C. Chicone)
}

AbStract. We consider an equation

$$
-y^{\prime \prime}(x)+q(x) y(x)=f(x), \quad x \in R,
$$

where $f(x) \in L_{p}(R), p \in[1, \infty]\left(\|f\|_{\infty}:=C(R)\right)$, and $0 \leq q(x) \in L_{1}^{\text {loc }}(R)$. By a solution of equation (1), we mean any function $y(x)$ such that $y(x), y^{\prime}(x) \in$ $A C^{\text {loc }}(R)$, and equality (1) holds almost everywhere on $R$. In this paper, we obtain a criterion for the correct solvability of $(1)$ in $L_{p}(R), p \in[1, \infty]$.

\section{$\S 1$. INTRODUCTION}

In this paper, we consider a Sturm-Liouville equation

$$
-y^{\prime \prime}(x)+q(x) y(x)=f(x), \quad x \in R,
$$

where $f(x) \in L_{p}(R), p \in[1, \infty]\left(\|f\|_{\infty}:=C(R)\right)$, and

$$
0 \leq q(x) \in L_{1}^{\text {loc }}(R) .
$$

By a solution of equation (1.1), we mean any function $y(x)$ such that $y(x), y^{\prime}(x) \in$ $A C^{\mathrm{loc}}(R)$, and equality (1.1) holds almost everywhere on $R$. Throughout this section, we assume all of the above conventions are fulfilled. Our goal is to find the requirement for $q(x)$ which guarantees the correct solvability of (1.1) in the given space $L_{p}(R), p \in[1, \infty]$. We say that equation $(1.1)$ is correctly solvable in $L_{p}(R)$ if assertions I) and II) hold:

I) for every $f(x) \in L_{p}(R)$ there is a unique solution of (1.1) $y(x) \in L_{p}(R)$;

II) there is an absolute positive constant $c(p)$ such that the solution of (1.1) $y(x) \in L_{p}(R)$ satisfies the inequality

$$
\|y\|_{p} \leq c(p)\|f\|_{p}, \quad f(x) \in L_{p}(R) .
$$

Let us emphasize that in I) and II) there are no additional requirements to $y(x)$ (as, for example, some boundary conditions on $\pm \infty$ (see [1, 2]), or specific representation of $y(x)$ (see [3])). Thus I) and II) hold or fail depending only on the properties of $q(x)$. In particular, if one replaces (1.2) by the stronger condition (1.4),

$$
0<\varepsilon \leq q(x) \in L_{1}^{\text {loc }}(R), \quad \varepsilon=\text { const },
$$

Received by the editors April 6, 2000 and, in revised form, October 4, 2000.

2000 Mathematics Subject Classification. Primary 34C11, 34B40, 47E05.

Key words and phrases. Correct solvability, Sturm-Liouville equation.

This research was supported by the Israel Academy of Sciences under Grant 431/95.

(C)2001 American Mathematical Society 
then equation (1.1) is correctly solvable in $L_{p}(R)$ for all $p \in[1, \infty]$ (see Theorem 1.2 below). Therefore, the question of whether I) and II) hold only arises when the graph of $q(x)$ is not separated from the number axis; for example, as in the cases a), b), c):
a) $q(x)=e^{|x|}+e^{|x|} \sin e^{|x|}$
b) $\quad q(x)=2 \cos ^{2} 2^{-1}|x|^{\theta}, \theta>0$;
c) $q(x)=\left(1+x^{2}\right)^{-1}+\theta\left(1+x^{2}\right)^{-1} \cos e^{|x|}, \quad|\theta| \leq 1$.

We now present the main results of the paper, Theorems 1.1 and 1.2.

First define auxiliary functions $d(x)$ and $q^{*}(x)$. We temporarily suppose that together with $(1.2), q(x)$ also satisfies (1.6):

$$
\int_{-\infty}^{x} q(t) d t>0, \quad \int_{x}^{\infty} q(t) d t>0 \quad \text { for all } \quad x \in R .
$$

Fix $x \in R$ and consider equation (1.7) in $d \geq 0$;

$$
F(d)=2, \quad F(d) \stackrel{\text { def }}{=} d \int_{x-d}^{x+d} q(\xi) d \xi
$$

For every $x \in R$ equation (1.7) has a unique finite positive solution [4]. Let $d(x)$ be the solution of (1.7) for $x \in R, q^{*}(x) \stackrel{\text { def }}{=} d^{-2}(x)$. The function $q^{*}(x)$ is continuous for $x \in R$ [4] and is a Steklov type average [5] with step $d(x)$ for the function $q(t)$ at point $t=x$, because (1.7) immediately implies

$$
q^{*}(x)=\frac{1}{d^{2}(x)}=\frac{1}{2 d(x)} \int_{x-d(x)}^{x+d(x)} q(t) d t, \quad x \in R .
$$

The functions $d(x), q^{*}(x)$ were introduced by M.O. Otelbaev [7].

Theorem 1.1. Equation (1.1) is correctly solvable in $L_{p}(R), p \in[1, \infty]$, if and only if condition (1.6) and (1.9) hold together:

$$
q_{0}^{*}>0, \quad q_{0}^{*} \stackrel{\text { def }}{=} \inf _{x \in R} q^{*}(x)
$$

In particular, one of the following assertions A) and B) holds:

A) for all $p \in[1, \infty]$ equation (1.1) is correctly solvable in $L_{p}(R)$;

B) for all $p \in[1, \infty]$ equation $(1.1)$ is not correctly solvable in $L_{p}(R)$.

Note the following feature of Theorem 1.1 which is important for its applications: the function $q^{*}(x)$ can be computed only for some particular $q(x)$.

At the same time, in order to apply Theorem 1.1, instead of the values of $q^{*}(x)$, it suffices to know their two-sided estimates (sharp by order). Such inequalities can be obtained for a sufficiently large class of functions $q(x)$ (see $\S 4$ ). Moreover, a useful complement to Theorem 1.1 is Theorem 1.2, which is equivalent to Theorem 1.1 . 
Theorem 1.2. Let $p \in[1, \infty]$ be given. Equation (1.1) is correctly solvable in $L_{p}(R)$ if and only if there is $a \in(0, \infty)$ such that

$$
q_{0}(a)>0, \quad q_{0}(a) \stackrel{\text { def }}{=} \inf _{x \in R} \int_{x-a}^{x+a} q(t) d t .
$$

Corollary 1.2.1. Let $q(x) \in L_{1}(-\infty, 0)$ or $q(x) \in L_{1}(0, \infty)$. Then for all $p \in$ $[1, \infty]$, equation (1.1) is not correctly solvable in $L_{p}(R)$.

See $\S 4$ for more details concerning the techniques for application of Theorems 1.1 and 1.2, where we study equations (1.1) with coefficients (1.5). Finally, we stress that the equivalent Theorems 1.1 and 1.2 are different in what concerns applications.

Criterion (1.10) is expressed in terms of the function $q(x)$ and not of its average $q^{*}(x)$ (see (1.9)), and is therefore more convenient for checking correct solvability in $L_{p}(R)$ of concrete equations (1.1) (see $\left.\S 4\right)$. At the same time, criterion (1.9) is applied more often than (1.10) when studying general properties of the solutions of (1.1) or objects related to (1.1). See proof of the "sufficiency part" for Theorem 1.1 in $\S 2$ and [1], [2], 3], 4], [5] for more details.

\section{§2. Proof of the First Criterion}

In this section, we prove Theorem 1.1 (the first criterion). Throughout the sequel, we denote by $c$ absolute positive constants which are not essential for exposition and may differ even within a single chain of computations.

I. Proof of Theorem 1.1 for $p \in[1, \infty)$. Necessity. For some $p \in[1, \infty)$, let equation (1.1) be correctly solvable in $L_{p}(R)$. To verify inequalities (1.6), assume the contrary: let one of the integrals (1.6) be zero for some $x=x_{0}$. Set $x_{0}=0$,

$$
\int_{0}^{\infty} q(t) d t=0 .
$$

Let us introduce a cutting function $\varphi(x) \in C_{0}^{\infty}(R)$ such that $\operatorname{supp} \varphi(x)=\left[-\frac{1}{4}, \frac{1}{4}\right]$, $\varphi(x)=1$ for $|x| \leq \frac{1}{8}$ and

$$
0 \leq \varphi(x) \leq 1 \quad \text { for } \quad \frac{1}{8} \leq|x| \leq \frac{1}{4}
$$

Denote $\tau_{k}=\left\|\varphi^{(k)}(x)\right\|_{C(R)}, \quad k=1,2$, and $\tau=\tau_{1}+\tau_{2}$.

Let us also introduce functions $\varphi_{n}(x), n=1,2, \ldots, y_{0}(x)$, and a set $U$ :

$$
\begin{aligned}
\varphi_{n}(x)=\varphi\left(\frac{x-n^{6}}{n^{2}}\right), n & =1,2, \ldots, \quad y_{0}(x)=\sum_{n=1}^{\infty} \varphi_{n}(x), x \in R, \\
U & =\bigcup_{n=1}^{\infty} \operatorname{supp} \varphi_{n}(\cdot) .
\end{aligned}
$$

Clearly, $\operatorname{supp} \varphi_{n}(\cdot)=\left[n^{6}-\frac{n^{2}}{4}, n^{6}+\frac{n^{2}}{4}\right]$ and $\operatorname{supp} \varphi_{n}(\cdot) \cap \operatorname{supp} \varphi_{m}(\cdot)=\emptyset$ for $n \neq$ $m$. Therefore, in (2.3), the sum representing $y_{0}(x)$ contains at most one nonzero 
summand or equals zero. Hence

$$
y_{0}^{\prime \prime}(x)=\sum_{n=1}^{\infty} \varphi_{n}(x)^{\prime \prime}=\sum_{n=1}^{\infty}\left[\varphi\left(\frac{x-n^{6}}{n^{2}}\right)\right]^{\prime \prime} \stackrel{\text { def }}{=}-f_{0}(x), x \in R .
$$

From (2.1) and (1.2), it follows that $q(x)=0$ almost everywhere on $R_{+}$. Hence almost everywhere on $R$ we have

$$
-y_{0}^{\prime \prime}(x)+q(x) y_{0}(x)=f_{0}(x), x \in R .
$$

In (2.5), $f_{0}(x) \in L_{p}(R)$ since (2.4) implies

$$
\left\|f_{0}\right\|_{p}^{p}=\sum_{n=1}^{\infty}\left\|\varphi_{n}^{\prime \prime}\right\|_{p}^{p} \leq c \tau^{p} \sum_{n=1}^{\infty} \frac{n^{2}}{n^{4 p}}<\infty .
$$

Since (1.1) is correctly solvable in $L_{p}(R)$, there is a solution of $(2.5), z_{0}(x) \in L_{p}(R)$. Since $q(x)=0$ almost everywhere on $R_{+}$, we have

$$
\left.\begin{array}{l}
-y_{0}^{\prime \prime}(x)=f_{0}(x), x \in R_{+} \\
-z_{0}^{\prime \prime}(x)=f_{0}(x), x \in R_{+}
\end{array}\right\} \Rightarrow\left\{\begin{array}{l}
z_{0}(x)=y_{0}(x)+c_{1}+c_{2} x, x \in R_{+} \\
c_{1}=\text { const, } c_{2}=\text { const } .
\end{array}\right.
$$

But $y_{0}(x) \equiv 0$ for $x \in R_{+} \backslash U$, and therefore

$$
z_{0}(x)=c_{1}+c_{2} x, \quad x \in R_{+} \backslash U .
$$

Assuming that in (2.7) $\left|c_{1}\right|+\left|c_{2}\right| \neq 0$, we get a contradiction:

$$
\begin{aligned}
\infty & >\left\|z_{0}\right\|_{p}^{p} \geq \sum_{n=1}^{\infty} \int_{n^{6}+4^{-1} n^{2}}^{n^{6}+4^{-1} n^{2}+1}\left|z_{0}(t)\right|^{p} d t \\
& =\sum_{n=1}^{\infty} \int_{n^{6}+4^{-1} n^{2}}^{n^{6}+4^{-1} n^{2}+1}\left|c_{1}+c_{2} x\right|^{p} d x=\infty .
\end{aligned}
$$

Hence $c_{1}=c_{2}=0 \Rightarrow y_{0}(x)=z_{0}(x), x \in R_{+}$. But then

$$
\infty>\left\|f_{0}\right\|_{p}^{p} \geq c(p)\left\|z_{0}\right\|_{p}^{p}=c(p)\left\|y_{0}\right\|_{p}^{p} \geq c(p) \sum_{n=1}^{\infty} \int_{n^{6}-8^{-1} n^{2}}^{n^{6}+8^{-1} n^{2}} 1 d t=\infty,
$$

a contradiction. Hence inequalities (1.6) hold. Let us now check (1.9). Fix $x \in R$ and consider a Cauchy problem

$$
\begin{gathered}
y^{\prime \prime}(t)=q(t) y(t), t \in R, \\
\left.y(t)\right|_{t=x}=1,\left.\quad y^{\prime}(t)\right|_{t=x}=0 .
\end{gathered}
$$

We need some auxiliary assertions.

Lemma 2.1. Under conditions (1.2) and (1.6), the solution $y(t)$ of problem (2.8) - (2.9) satisfies the following relations:

$$
\begin{gathered}
y(t) \geq 1, \quad \operatorname{sign} y^{\prime}(t)=\operatorname{sign}(t-x) \quad \text { for } \quad t \in R, \\
\frac{y(x+t)+y(x-t)}{2}=1+\frac{1}{2} \int_{0}^{t} \int_{x-s}^{x+s} q(\xi) y(\xi) d \xi d s, \quad t \geq 0,
\end{gathered}
$$




$$
\begin{aligned}
& 1 \leq y(t) \leq 4 \quad \text { for } \quad t \in\left[x-\frac{d(x)}{4}, x+\frac{d(x)}{4}\right] \\
& \left|y^{\prime}(t)\right| \leq \frac{8}{d(x)} \quad \text { for } \quad t \in\left[x-\frac{d(x)}{4}, x+\frac{d(x)}{4}\right] .
\end{aligned}
$$

Proof. Relations (2.10) can be viewed as a particular case of Lemma 3.1 of [4]. To prove (2.11), one has to integrate (2.8) twice: first over $[x-s, x+s], s \geq 0$, then by $s \in[0, t], t \geq 0$; one of them has to use (2.9). Consider (2.12). As already mentioned in $\S 1$, under conditions (1.2) and (1.6) the function $d(x)$ exists and is defined uniquely for all $x \in R$. Let

$$
M(t)=\max \{y(x+t), y(x-t)\}, t \geq 0 .
$$

Then for $t \in\left[0, \frac{d(x)}{4}\right]$ from (2.11), (2.10) and (1.7), we obtain

$$
\begin{aligned}
\frac{M(t)}{2} & <\frac{y(x+t)+y(x-t)}{2}=1+\frac{1}{2} \int_{0}^{t} \int_{x-s}^{x+s} q(\xi) y(\xi) d \xi d s \\
& \leq 1+\frac{M(t)}{2} \cdot t \int_{x-t}^{x+t} q(\xi) d \xi \leq 1+\frac{M(t)}{8}\left[d(x) \int_{x-\frac{d(x)}{4}}^{x+\frac{d(x)}{4}} q(\xi) d \xi\right] \\
& \leq 1+\frac{M(t)}{8}\left[d(x) \int_{x-d(x)}^{x+d(x)} q(\xi) d \xi\right]=1+\frac{M(t)}{4} \Rightarrow(2.11) .
\end{aligned}
$$

To prove (2.13), we integrate (2.8) over $[x, t], x \leq t$ taking into account (2.9), we obtain $y^{\prime}(t)$; the estimate for $\left|y^{\prime}(t)\right|$ now follows from (2.12) and (1.7):

$$
\begin{aligned}
y^{\prime}(t) & =\int_{x}^{t} q(\xi) y(\xi) d \xi, x \leq t \\
& \Rightarrow\left|y^{\prime}(t)\right| \leq 4 \int_{x}^{t} q(\xi) d \xi \leq 4 \int_{x-d(x)}^{x+d(x)} q(\xi) d \xi=\frac{8}{d(x)} .
\end{aligned}
$$

Inequality (2.13) for $t \in[x-d(x), x]$ can be proved in a similar way.

Turn to (1.9). Fix $x \in R$ and introduce a function

$$
z(t)=d(x)^{2-1 / p} \varphi\left(\frac{t-x}{d(x)}\right) y(t), t \in R .
$$

Here $y(t)$ is the solution of $(2.8)-(2.9)$. A straightforward computation shows that $z(t)$ is a solution of $(2.15)$ with the right-hand side $f_{1}(t)$ given in $(2.16)$ :

$$
-z^{\prime \prime}(t)+q(t) z(t)=f_{1}(t), x \in R,
$$

$$
f_{1}(t)=-d(x)^{2-1 / p}\left[\varphi\left(\frac{t-x}{d(x)}\right)\right]^{\prime \prime} y(t)-2 d(x)^{2-1 / p}\left[\varphi\left(\frac{t-x}{d(x)}\right)\right]^{\prime} y^{\prime}(t), t \in R .
$$


Below we estimate the norms $\left\|f_{1}\right\|_{p}$ and $\|z\|_{p}$ using Lemma 2.1 and the property of $\varphi(\cdot)$ :

$$
\begin{aligned}
\left\|f_{1}\right\|_{p}^{p}= & \int_{x-\frac{d(x)}{4}} \mid f_{1}(t)^{p} d t \leq c\left\{\int_{x-\frac{d(x)}{4}} d(x)^{2 p-1}\left|\left[\varphi\left(\frac{d-x}{d(x)}\right)\right]^{\prime \prime}\right|^{p}|y(t)|^{p} d t\right. \\
+ & \left.\int_{x-\frac{d(x)}{4}}^{x+\frac{d(x)}{4}} d(x)^{2 p-1}\left|\left[\varphi\left(\frac{t-x}{d(x)}\right)\right]^{\prime}\right|^{p}\left|y^{\prime}(t)\right|^{p} d t\right\} \\
\leq & c d(x)^{2 p-1}\left\{\int_{x-\frac{d(x)}{4}}\left(4 \tau_{2}\right)^{p} \frac{d t}{d(x)^{2 p}}+\int_{x-\frac{d(x)}{4}}^{x+\frac{d(x)}{4}}\left(8 \tau_{1}\right)^{p} \frac{d t}{d(x)^{2 p}}\right\} \leq c<\infty \\
\text { 7) } & \quad\|z\|_{p}^{p}=\int_{x-\frac{d(x)}{4}} d(x)^{2 p-1}\left|\varphi\left(\frac{t-x}{d(x)}\right)\right|^{p}|y(t)|^{p} d t \leq c d(x)^{2 p}<\infty .
\end{aligned}
$$

From $(2.17)-(2.18)$ it follows that $z(t), f_{1}(t) \in L_{p}(R)$. Then, since equation (1.1) is correctly solvable in $L_{p}(R)$, we have inequality (1.3) with $y(t)=z(t)$, $f(t)=f_{1}(t), t \in R$. Now, in addition to (2.18), we estimate $\|z\|_{p}$ from below. By Lemma 3.1 and the definition of $\varphi(\cdot)$, for $t \in\left[x-\frac{d(x)}{8}, x+\frac{d(x)}{8}\right]$, we obtain the following relations:

$$
\begin{aligned}
& z(t)=d(x)^{2-1 / p} \varphi\left(\frac{t-x}{d(x)}\right) y(t) \equiv d(x)^{2-1 / p} y(t) \geq d(x)^{2-1 / p} \\
& \Rightarrow\|z\|_{p}^{p} \geq \int_{x-\frac{d(x)}{8}}^{x+\frac{d(x)}{8}}|z(t)|^{p} d t \geq \frac{1}{4} d(x)^{2 p} .
\end{aligned}
$$

Now, (2.19) and (1.30) imply

$$
c^{-1} d^{2}(x) \leq\|z\|_{p} \leq c(p)\left\|f_{1}\right\|_{p} \Rightarrow d(x)^{2} \leq c_{1}(p), x \in R .
$$

From (2.20) it follows that $q^{*}(x)=d(x)^{-2} \geq c^{-1}, x \in R$.

Remark. Test function (2.14) was first used in [8] with another purpose (see also 7. ch.VII, §7].

II. Proof of Theorem 1.1 for $p=\infty$. Necessity. Let equation (1.1) be correctly solvable in $C(R)$. To verify inequalities (1.6), assume the contrary: for some $x=x_{0}$ one of the integrals (1.6) equals zero. Suppose that (2.1) holds. Let us introduce functions $\psi_{n}(x), n=1,2, \ldots, \tilde{y}_{0}(x)$, and a set $\tilde{U}_{0}$

$$
\begin{gathered}
\psi_{n}(x)=n^{2} \varphi\left(\frac{x-n^{6}}{n^{2}}\right), n=1,2, \ldots ; \quad \tilde{y}_{0}(x)=\sum_{n=1}^{\infty} \psi_{n}(x), x \in R, \\
\tilde{U}_{0}=\bigcup_{n=1}^{\infty} \operatorname{supp} \psi_{n}(\cdot) .
\end{gathered}
$$


Then, as in I, we obtain (2.22) and (2.23):

$$
\begin{gathered}
\tilde{y}_{0}^{\prime \prime}(x)=\sum_{n=1}^{\infty} \psi_{n}^{\prime \prime}(x)=\sum_{n=1} n^{2}\left[\varphi\left(\frac{x-n^{6}}{n^{2}}\right)\right]^{\prime \prime}:=-\tilde{f}_{0}(x), x \in R, \\
-\tilde{y}_{0}^{\prime \prime}(x)+q(x) \tilde{y}_{0}(x)=\tilde{f}_{0}(x), x \in R .
\end{gathered}
$$

Clearly, $\tilde{f}_{0}(x) \in C(R)$ since

$$
\left\|\tilde{f}_{0}\right\|_{C(R)}=\sup _{n} n^{2}\left\|\left[\varphi\left(\frac{x-n^{6}}{n^{2}}\right)\right]^{\prime \prime}\right\|_{C(R)} \leq \tau_{2}<\infty .
$$

Hence there exists a solution $z_{0}(x) \in C(R)$ of equation (1.1) with the right-hand side $\tilde{f}_{0}(x), x \in R$. Then

$$
\left.\begin{array}{l}
-y_{0}^{\prime \prime}(x)+q(x) \tilde{y}_{0}(x)=\tilde{f}_{0}(x), x \in R \\
-z_{0}^{\prime \prime}(x)+q(x) z_{0}(x)=\tilde{f}_{0}(x), x \in R
\end{array}\right\} \Rightarrow\left\{\begin{array}{l}
\tilde{y}_{0}(x)=z_{0}(x)+c_{0}+c_{1} x \\
x \in R .
\end{array}\right.
$$

In (2.24), $c_{0}, c_{1}$ are constants. Since $\tilde{y}_{0}(x) \equiv 0$ for $x \in R \backslash \tilde{U}_{0}$ and $\left\|z_{0}\right\|_{C(R)}<\infty$, we have $c_{1}=0$. Furthermore, for $x_{n}=n^{6}$ from (2.24), it follows that

$$
\tilde{y}_{0}(x)=n^{2}=z_{0}\left(n^{6}\right)+c_{0} \Rightarrow 1=\frac{z_{0}\left(n^{6}\right)+c_{0}}{n^{2}} \leq \frac{\left\|z_{0}\right\|_{C(R)}+\left|c_{0}\right|}{n^{2}},
$$

a contradiction. Hence inequalities (1.6) hold.

Now to prove (1.9) for $p=\infty$, one must repeat, word for word, the corresponding argument from $\mathbf{I}$.

III. Proof of Theorem 1.1. Sufficiency. To prove the "sufficiency part" of Theorem 1.1, we use assertions from [4], 6]. Let us introduce necessary definitions and facts.

Theorem 2.1 (4]). Suppose that (1.6) holds. Then there exists a fundamental system of solutions $\{u(x), v(x)\}$ of the equation

$$
z^{\prime \prime}(x)=q(x) z(x), \quad x \in R,
$$

such that

$$
\begin{gathered}
u(x)>0, \quad v(x)>0, \quad u^{\prime}(x) \leq 0, \quad v^{\prime}(x) \geq 0 \quad \text { for } \quad x \in R, \\
v^{\prime}(x) u(x)-u^{\prime}(x) v(x)=1 \quad \text { for } \quad x \in R, \\
\lim _{x \rightarrow-\infty} \frac{v(x)}{u(x)}=\lim _{x \rightarrow \infty} \frac{u(x)}{v(x)}=0 .
\end{gathered}
$$

Let $G(x, t), x, t \in R$, be the Green's function

$$
G(x, t)= \begin{cases}u(x) \cdot v(t), & x \geq t, \\ u(t) \cdot v(x), & x \leq t,\end{cases}
$$

corresponding to (1.1).

Theorem 2.2 ([6]). For $G(x, t)$ one has the following representation:

$$
G(x, t)=\sqrt{\rho(x) \rho(t)} \exp \left(-\frac{1}{2}\left|\int_{x}^{t} \frac{d \xi}{\rho(\xi)}\right|\right)
$$

where $x, t \in R, \rho(x)=\left.G(x, t)\right|_{t=x}=u(x) v(x)$. 
Remark. For the proof of (2.28) under conditions (1.2) and (1.6), see [4].

Theorem 2.3 (4]). Suppose that (1.6) holds. Then for $x \in R$ one has inequalities

$$
\frac{d(x)}{4} \leq \rho(x) \leq \frac{3}{2} d(x)
$$

(see (1.7)).

Theorem 2.4 (4]). Suppose that (1.6) holds. Then (2.25) has no solutions $z(x) \in$ $L_{p}(R), p \in[1, \infty]$, apart from $z(x) \equiv 0$. If, moreover,

$$
H<\infty, \quad H \stackrel{\text { def }}{=} \sup _{x \in R} \int_{-\infty}^{\infty} G(x, t) d t
$$

then assertions I) and II) from $\S 1$ hold.

Thus, according to Theorem 2.4, in order to prove Theorem 1.1 it is sufficient to check that the inequality (2.30) is fulfilled subject to (1.6) and (1.9). The latter follows from the relations

$$
\begin{aligned}
H & =\sup _{x \in R} \int_{-\infty}^{\infty} G(x, t) d t=\sup _{x \in R} \int_{-\infty}^{\infty} \sqrt{\rho(x) \rho(t)} \exp \left(-\frac{1}{2}\left|\int_{x}^{t} \frac{d \xi}{\rho(\xi)}\right|\right) d t \\
& \leq \frac{3}{2 q_{0}^{*}} \sup _{x \in R} \int_{-\infty}^{\infty} \exp \left(-\frac{1}{3} \sqrt{q_{0}^{*}}|t-x|\right) d t=\frac{9}{\left(q_{0}^{*}\right)^{3 / 2}}<\infty,
\end{aligned}
$$

which are based on (2.18) and (2.29).

\section{§3. Proof of the SECOND CRITERION}

This section contains a proof of Theorem 1.2 (second criterion) which is based on Theorem 1.1. Below we also use Lemma 3.1.

Lemma 3.1. Suppose that (1.6) holds. The inequality $\eta \geq d(x)(0 \leq \eta \leq d(x))$ holds if and only if

$$
2 \leq \eta \int_{x-\eta}^{x+\eta} q(t) d t \quad\left(2 \geq \eta \int_{x-\eta}^{x+\eta} q(t) d t\right) .
$$

Proof. Necessity. Let $\eta \geq d(x)$. Then $[x-d(x), x+d(x)] \subseteq[x-\eta, x+\eta]$, and therefore

$$
2=d(x) \int_{x-d(x)}^{x+d(x)} q(t) d t \leq \eta \int_{x-\eta}^{x+\eta} q(t) d t .
$$

Sufficiency. Let (3.1) hold. Assume the contrary: $\eta<d(x)$. Then $[x-\eta, x+\eta] \subset$ $[x-d(x), x+d(x)]$, and therefore

$$
2 \leq \eta \int_{x-\eta}^{x+\eta} q(t) d t<d(x) \int_{x-d(x)}^{x+d(x)} q(t) d t=2,
$$

a contradiction. 
Proof of Theorem 1.2. Necessity. Suppose that equation (1.1) is correctly solvable in $L_{p}(R), p \in[1, \infty]$. Then by Theorem 1.1, conditions (1.6) and (1.9) hold. This implies that $q_{0}(a)>0$ for some $a>0$. Let us prove this ad absurdum. Thus let $q_{0}(a)=0$ for every $a>0$. Fix a sequence $\left\{a_{n}\right\}_{n=1}^{\infty}$ such that $0<a_{1}<a_{2}<$ $\cdots<a_{n}<\ldots, \lim _{n \rightarrow \infty} a_{n}=\infty$. Then for every $n=1,2, \ldots$, there is $x_{n}$ such that

$$
a_{n} \int_{x_{n}-a_{n}}^{x_{n}+a_{n}} q(t) d t \leq 2 .
$$

Let us now fix some $\varepsilon>0$. Clearly, $a_{n_{0}} \geq \varepsilon^{-1}$ for some $n_{0}=n_{0}(\varepsilon)$. Then by (3.2),

$$
\frac{1}{\varepsilon} \int_{x_{n_{0}}-\varepsilon^{-1}}^{x_{n_{0}}+\varepsilon^{-1}} q(t) d t \leq a_{n_{0}} \int_{x_{n_{0}}-a_{n_{0}}}^{x_{n_{0}}+a_{n_{0}}} q(t) d t \leq 2 .
$$

By (3.3) and Lemma 3.1, we get

$$
d\left(x_{n_{0}}\right) \geq \varepsilon^{-1} \Rightarrow \varepsilon^{2} \geq q^{*}\left(x_{n_{0}}\right) \Rightarrow q_{0}^{*}=0
$$

(see (1.9)), a contradiction.

Proof of Theorem 1.2. Sufficiency. Let $q_{0}\left(a_{0}\right)>0$ for some $a_{0}>0$. Then, clearly, (1.6) holds. We choose $a$, satisfying conditions $a \geq a_{0}, a q_{0}\left(a_{0}\right) \geq 2$. Then the following relations hold:

$$
a \int_{x-a}^{x+a} q(t) d t \geq a \int_{x-a_{0}}^{x+a_{0}} q(t) d t \geq a q_{0}\left(a_{0}\right) \geq 2, x \in R .
$$

From (3.4) and Lemma 3.1 we conclude that $d(x) \leq a$ for $x \in R$. Then $q^{*}(x) \geq a^{-2}$ for $x \in R$ and therefore $q_{0}^{*} \geq a^{-2}>0$. It remains to use Theorem 1.1.

\section{$\S 4$. EXAMPLES}

In this section, we consider equations (1.1) with coefficients (1.5).

Example (1.5a). Application of Theorem 1.1. As we mentioned (see $\S 1$ ), to apply Theorem 1.1, we need some additional information on properties of $q^{*}(x)$. Such information can be derived from assertions such as Theorem 4.1.

Theorem 4.1. Let (1.6) hold and assume that $q(x)$ can be represented in the form $q(x)=q_{1}(x)+q_{2}(x), x \in R$, where $q_{1}(x)$ is continuous for $x \in R, q_{1}(x)>0$ for $x \in R, q_{2}(x) \in L_{1}^{\mathrm{loc}}(R)$. Let $A(x)=\left[0,2 q_{1}(x)^{-1 / 2}\right]$ and

$$
\begin{gathered}
h_{1}(x)=\frac{1}{\sqrt{q_{1}(x)}} \sup _{t \in A(x)}\left|\int_{0}^{t}\left[q_{1}(x+s)-2 q_{1}(x)+q_{1}(x-s)\right] d s\right|, \\
h_{2}(x)=\frac{1}{\sqrt{q_{1}(x)}} \sup _{t \in A(x)}\left|\int_{x-t}^{x+t} q_{2}(s) d s\right| .
\end{gathered}
$$

If $h_{1}(x) \rightarrow 0, h_{2}(x) \rightarrow 0$ as $|x| \rightarrow \infty$, then

$$
c^{-1} q_{1}(x) \leq q^{*}(x) \leq c q_{1}(x) \quad \text { for } \quad x \in R .
$$


Remark. Theorem 4.1 was obtained in [5] under an additional assumption: $\left.q_{(} x\right) \geq 1$ for $x \in R$. Its proof (see [5]) is also valid under the assumptions made above.

To apply Theorem 4.1 to Example (1.5a), we set $q_{1}(x)=e^{|x|}, q_{2}(x)=e^{|x|} \sin e^{|x|}$. Since the functions $q_{1}(x), q_{2}(x)$ are even, below we only consider the case $x \geq 0$. Let us verify that $h_{1}(x), h_{2}(x)$ tend to zero as $x \rightarrow \infty$.

Since $q_{1}(x) \geq 1$ for $x \geq 0$, we have $A(x) \subseteq[0,2]$. Therefore, the following Taylor expansions converge for all $x \geq 0$ :

$$
\begin{gathered}
h_{1}(x)=e^{-x / 2} \sup _{t \in A(x)}\left|\int_{0}^{t}\left[e^{x+s}-2 e^{x}+e^{x-s}\right] d s\right|=e^{x / 2} \sup _{t \in A(x)}\left|\int_{0}^{t}\left[e^{s}+e^{-s}-2\right] d s\right| \\
=e^{x / 2} \sup _{t \in A(x)}\left|\int_{0}^{t}\left[s^{2}+\frac{s^{4}}{12}+\ldots\right] d s\right| \leq \frac{c}{e^{x}} \rightarrow 0 \text { for } x \rightarrow \infty, \\
h_{2}(x)=\frac{1}{e^{x / 2}} \sup _{t \in A(x)}\left|\int_{x-t}^{x+t} e^{s} \sin e^{s} d s\right| \leq \frac{c}{e^{x / 2}} \rightarrow 0 \text { for } x \rightarrow \infty .
\end{gathered}
$$

Hence (4.3) holds. In our particular case, we can rewrite it as follows:

$$
c^{-1} e^{|x|} \leq q^{*}(x) \leq c e^{|x|}, x \in R .
$$

From (4.4) we obtain $q_{0}^{*}>0$ (see (1.9)). Therefore, by Theorem 1.1, equation (1.1) with coefficient $(1.5 \mathrm{a})$ is correctly solvable in $L_{p}(R)$ for all $p \in[1, \infty]$.

Example (1.5a). Application of Theorem 1.2. Let $a \gg 1$. Then we have the following obvious relations:

$$
\begin{aligned}
& \inf _{x \leq 0} \int_{x-a}^{x+a}\left[e^{-t}+e^{-t} \sin e^{-t}\right] d t=\inf _{x \geq 0} \int_{x-a}^{x+a}\left[e^{t}+e^{t} \sin e^{t}\right] d t \\
& \quad=\inf _{x \geq 0} e^{x+a}\left[1-e^{-2 a}-\frac{\cos e^{x+a}-\cos e^{x-a}}{e^{x+a}}\right] \geq \frac{1}{2} .
\end{aligned}
$$

Hence $q_{0}(a) \geq \frac{1}{2}$ for $a \gg 1($ see $(1.10))$, and it remains to apply Theorem 1.2.

Example (1.5b). Let $\theta \geq 1$. For $x \geq 2 a, a \gg 1$, we get

$$
\begin{aligned}
\int_{x-a}^{x+a}\left(1+\cos t^{\theta}\right) d t & =2 a+\frac{1}{\theta} \int_{x-a}^{x+a} \frac{\theta t^{\theta-1} \cos t^{\theta} d t}{t^{\theta-1}} \\
& =2 a+\left.\frac{1}{\theta} \frac{\sin t^{\theta}}{t^{\theta-1}}\right|_{x-a} ^{x+a}+\frac{\theta-1}{\theta} \int_{x-a}^{x+a} \frac{\sin t^{\theta}}{t^{\theta}} d t \\
& \geq 2 a-\frac{1}{(3 a)^{\theta-1}}-\frac{1}{a^{\theta-1}}-\frac{2 a}{a^{\theta}} \geq 2 a\left[1-\frac{2}{a}\right] \geq \frac{1}{2}
\end{aligned}
$$

Furthermore, the function $F(x)=\int_{x-a}^{x+a}\left(1+\cos |t|^{\theta}\right) d t$ is continuous and positive on $[0,2 a]$, and therefore it attains its minimum $F_{0}>0$. Then $F(x) \geq \min \left\{\frac{1}{2}, F_{0}\right\}>0$ for $x \geq 0$. 
The case $x \leq 0$ can be considered in a similar way. Hence for $\theta \geq 1$, (1.10) holds and equation (1.1) is correctly solvable in $L_{p}(R)$ for all $p \in[1, \infty]$. Now let $\theta \in(0,1)$. Set $x_{n}=[(2 n+1) \pi]^{1 / \theta}, n=1,2, \ldots$ For any $a \geq 0$, we get

$$
\begin{aligned}
J_{n}(a) & \stackrel{\text { def }}{=} \int_{x_{n}-a}^{x_{n}+a}\left(1+\cos t^{\theta}\right) d t=\int_{x_{n}-a}^{x_{n}}\left(1+\cos t^{\theta}\right) d t+\int_{x_{n}}^{x_{n}+a}\left(1+\cos t^{\theta}\right) d t \\
& =2 a+\int_{0}^{a}\left[\cos \left(x_{n}-z\right)^{\theta}+\cos \left(x_{n}+z\right)^{\theta}\right] d z \\
& =2 a-\int_{0}^{a}\left\{\cos \left[\left(x_{n}-z\right)^{\theta}-x_{n}^{\theta}\right]+\cos \left[\left(x_{n}+z\right)^{\theta}-x_{n}^{\theta}\right]\right\} d z .
\end{aligned}
$$

From the binomial Taylor expansion, it follows that

$$
\begin{aligned}
& \alpha_{n}(z) \stackrel{\text { def }}{=}\left(x_{n}+z\right)^{\theta}-x_{n}^{\theta}=\theta z x_{n}^{\theta-1}+O\left(x_{n}^{\theta-2)}\right), \\
& \beta_{n}(z) \stackrel{\text { def }}{=}\left(x_{n}-z\right)^{\theta}-x_{n}^{\theta}=-\theta z x_{n}^{\theta-1}+O\left(x_{n}^{\theta-2)}\right) .
\end{aligned}
$$

Clearly, $\alpha_{n}(z) \rightarrow 0, \beta_{n}(z) \rightarrow 0$ as $n \rightarrow \infty$ for any fixed $a \gg 1$. Therefore, using the Taylor expansion of cosine, we obtain from (4.5) and (4.6) - (4.7):

$$
\begin{aligned}
J_{n}(a) & =2 a-\int_{0}^{a}\left[2-\frac{\alpha_{n}^{2}(z)+\beta_{n}^{2}(z)}{2 !}+\frac{\alpha_{n}^{4}(z)+\beta_{n}^{4}(z)}{4 !}+\ldots\right] d z \\
& \leq c \int_{0}^{a}\left[\alpha_{n}^{2}(z)+\beta_{n}^{2}(z)\right] d z \leq \frac{c \theta^{2} a^{3}}{x_{n}^{2(1-\theta)}} \rightarrow 0 \text { for } n \rightarrow \infty .
\end{aligned}
$$

Hence by Theorem 1.2 , we conclude that for $\theta \in(0,1)$ equation (1.1) with coefficient (1.1) cannot be correctly solvable in $L_{p}(R)$ for all $p \in[1, \infty]$.

Example (1.5c). In this case, $q(x) \rightarrow 0$ as $|x| \rightarrow \infty$. Hence for any fixed $a>0$, we get

$$
\lim _{|x| \rightarrow \infty} \int_{x-a}^{x+a} q(t) d t=0 \Rightarrow \inf _{x \in R} \int_{x-a}^{x+a} q(t) d t=q_{0}(a)=0 .
$$

Therefore by Theorem 1.2, equation (1.1) with coefficient (1.5d) cannot be correctly solvable in $L_{p}(R)$ for all $p \in[1, \infty]$.

\section{REFERENCES}

1. N. Chernyavskaya and L. Shuster, Solvability in $L_{p}$ of the Dirichlet problem for a singular nonhomogeneous Sturm-Liouville equation, Methods and Applications of Analysis 5 (3) (1998), 259-272. MR 99m:34054

2. Solvability in $L_{p}$ of the Neumann problem for a singular nonhomogeneous SturmLiouville equation, to appear in Mathematika.

3. L Regularity of the inversion problem for the Sturm-Liouville equation in the spaces $L_{p}$, Methods and Applications of Analysis 7 (2000) no. 1, 65-84.

4. - Estimates for the Green function of a general Sturm-Liouville operator and their applications, Proc. Amer. Math. Soc. 127 (5) (1999), 1413-1426. MR 99h:34040

5. - Asymptotics on the diagonal of the Green function of a Sturm-Liouville operator and its applications, J. London Math. Soc. 61 (2000), 506-530. MR 2001b:34057 
6. E.B. Davies and E.M. Harrell, Conformally flat Riemannian metrics, Schrödinger operators and semiclassical approximation, J. Diff. Eq. 66 (1987), 165-188. MR 88a:35061

7. K. Mynbaev and M. Otelbaev, Weighted Function Spaces and the Spectrum of Differential Operators, Nauka, Moscow, 1988. MR 89h:46036

8. M. Otelbaev, On smoothness of solutions of differential equations, Izvestija Akad. Nauk Kazakh. SSR 5 (1977), 45-48. MR 58:17302

9. W.A. Steklov, Sur une méthode nouvelle pour résoudre plusiers problèmes sur le dévloppement d'une fonction arbitraire en séries infinies, Comptes Rendus, Paris 144 (1907), 1329-1332.

Department of Mathematics and Computer Science, Ben-Gurion University of the Negev, P.O.B. 653, BeER-Sheva, 84105, IsRAel

Department of Mathematics and Computer Science, Bar-Ilan University, Ramat-Gan, 52900, ISRAEL 